\title{
Growth of Generalize Iterated Entire Functions and Maximum Term
}

\author{
Ratan Kumar Dutta ${ }^{1}$, Nintu Mandal ${ }^{2}$ \\ ${ }^{1}$ Department of Mathematics, Netaji Mahavidyalaya, \\ Arambagh, Hooghly-712601, West Bengal, India \\ ${ }^{2}$ Department of Mathematics, Chandernagore College, \\ Chandernagore, Hooghly-712136, West Bengal, India \\ ratan_3128@yahoo.com,nintu311209@gmail.com
}

\begin{abstract}
In this article, we consider generalize relative iterations of entire functions and study comparative growth of the maximum term of generalize iterated entire functions with that of the maximum term of the related functions.
\end{abstract}

Keywords-Entire functions, maximum term, maximum modulus, iteration, order, lower order.

\section{INTRODUCTION, DEFINITIONS AND NOTATIONS}

Let $f(z)=\sum_{n=0}^{\infty} a_{n} z^{n}$ be an entire function defined in the open complex plane $C$. Then $M(r, f)=$ $\max _{|z|=r}|f(z)|$ and $\mu(r, f)=\max _{n}\left|a_{n}\right| r^{n}$ are respectively called the maximum modulus and maximum term of $f(z)$ on $|z|=r$. The following definitions are well known.

Definition 1.1 [9] The order $\rho_{f}$ and lower order $\lambda_{f}$ of a meromorphic function $f$ are defined as

$$
\rho_{f}=\limsup _{r \rightarrow \infty} \frac{\log \log M(r, f)}{\log r}
$$

and

$$
\lambda_{(f)}=\liminf _{r \rightarrow \infty} \frac{\log \log M(r, f)}{\log r} .
$$

Notation 1.2 [7] Let $\log ^{[m]} x=\log \left(\log ^{[m-1]} x\right)$ and $\exp ^{[m]} x=\exp \left(\exp ^{[m-1]} x\right)$ for positive integer $m$ where $\log ^{[0]} x=x$ and $\exp ^{[0]} x=x$.

A simple but useful relation between $M(r, f)$ and $\mu(r, f)$ is given in the following theorem.

Theorem 1.3 [8] Let $f$ be a non-constant entire function defined in the open complex plane $C$. Then for $0 \leq r<R$,

$$
\mu(r, f) \leq M(r, f) \leq \frac{R}{R-r} \mu(R, f)
$$

Taking $R=2 r$, for all sufficiently large values of $r$ we have

$$
\mu(r, f) \leq M(r, f) \leq 2 \mu(2 r, f)
$$

Taking two times logarithms in (1) it is easy to verify that

and

$$
\rho_{f}=\limsup _{r \rightarrow \infty} \frac{\log ^{[2]} \mu(r, f)}{\log r}
$$

$$
\lambda_{f}=\liminf _{r \rightarrow \infty} \frac{\log ^{[2]} \mu(r, f)}{\log r} .
$$

Definition 1.4 [1] Let $f$ and $g$ be two entire functions defined in the open complex plane $C$ and $d \in(0,1]$. Now we define

$$
f_{\text {and }}^{[n ; g]}(z)=(1-d) g^{[n-1 ; f]}(z)+d f\left(g^{[n-1 ; f]}(z)\right)
$$

$$
g^{[n ; f]}(z)=(1-d) f^{[n-1 ; g]}(z)+d g\left(f^{[n-1 ; g]}(z)\right),
$$

for any positive integer $n \geq 2$ where $f^{[1 ; g]}(z)=$ $(1-d) z+d f(z)$ and $g^{[1 ; f]}(z)=(1-d) z+d g(z)$.

From the above definition clearly all $f^{[n ; g]}$ and $g^{[n ; f]}$ are entire functions.

Recently Banerjee and Dutta [2] Dutta [4], [5] study some results on comparative growth properties of the maximum term of iterated entire functions.

In this paper, we study growth properties of the maximum term of generalize iterated entire functions as compared to the growth of the maximum term of the related function to generalize some earlier results. Throughout the paper we denote by $f(z), g(z)$ etc. non-constant entire functions of order (lower order) $\rho_{f},\left(\lambda_{f}\right), \rho_{g},\left(\lambda_{g}\right)$ etc. We do not explain the standard notations and definitions of the theory of entire functions as those are available in [6], [9] and [10].

\section{LEMMAS}

The following lemmas will be needed in the sequel.

Lemma 2.1 [3] For any two non-constant entire functions $f$ and $g$ defined in the open complex plane C, 


$$
\begin{gathered}
M\left(\frac{1}{8} M\left(\frac{r}{2}, g\right)-\mid\right. \\
\leq(0) \mid, f) \leq M(r, f \circ g) \\
\leq M(M(r, g), f),
\end{gathered}
$$

for all sufficiently large values of $r$.

Lemma 2.2 Let $f$ and $g$ be two non-constant entire functions such that $0<\lambda_{f} \leq \rho_{f}<\infty$ and $0<\lambda_{g} \leq$ $\rho_{g}<\infty$. Then for any $\varepsilon\left(0<\epsilon<\min \left\{\lambda_{f}, \lambda_{g}\right\}\right)$,

and

$$
\begin{aligned}
& \log { }^{[n]} M\left(r, f^{[n ; g]}\right) \\
& \geq\left(\lambda_{f}-\epsilon\right)(1+O(1)) \log M\left(\frac{r}{2^{n-1}}, g\right) \\
& +O(1), \text { when } n \text { is even }
\end{aligned}
$$

$$
\begin{aligned}
& \log ^{[n]} M\left(r, f^{[n ; g]}\right) \\
& \geq\left(\lambda_{g}-\epsilon\right)(1+O(1)) \log M\left(\frac{r}{2^{n-1}}, f\right) \\
& +O(1), \text { when } n \text { is odd, }
\end{aligned}
$$

for all sufficiently large values of $r$.

Proof. Let $\lambda_{f}$ and $\lambda_{g}$ be the lower orders of $f$ and $g$ respectively, then from Lemma 2.1 we have for all large values of $r$ and any $\varepsilon\left(0<\epsilon<\min \left\{\lambda_{f}, \lambda_{g}\right\}\right)$,

$$
\begin{aligned}
& M\left(r, f^{[n ; g]}\right) \\
& \geq M\left(r, f\left(g^{[n-1 ; f]}\right)\right)-M\left(r, g^{[n-1 ; f]}\right)+O(1) \\
& =(1+O(1)) M\left(r, f\left(g^{[n-1 ; f]}\right)\right) \\
& \geq(1+O(1)) M\left(\frac{1}{8} M\left(\frac{r}{2}, g^{[n-1 ; f]}\right)\right. \\
& \left.-\left|g^{[n-1 ; f]}(0)\right|, f\right) \\
& \geq(1+O(1)) M\left(\frac{1}{16} M\left(\frac{r}{2}, g^{[n-1 ; f]}\right), f\right) .
\end{aligned}
$$

Therefore

$$
\begin{aligned}
& \log M\left(r, f^{[n ; g]}\right) \\
& \geq \log M\left(\frac{1}{16} M\left(\frac{r}{2}, g^{[n-1 ; f]}\right), f\right)+O(1) \\
& \geq\left[\frac{1}{16} M\left(\frac{r}{2}, g^{[n-1 ; f]}\right)\right]^{\lambda_{f}-\epsilon} \\
& +O(1)
\end{aligned}
$$

$\log ^{[2]} M\left(r, f^{[n ; g]}\right)$

$\geq\left(\lambda_{f}-\epsilon\right) \log M\left(\frac{r}{2}, g^{[n-1 ; f]}\right)+\log \frac{1}{16}+O(1)$

$=\left(\lambda_{f}-\epsilon\right)(1+O(1)) \log M\left(\frac{r}{2}, g\left(f^{[n-2 ; g]}\right)+O(1)\right.$

$\geq\left(\lambda_{f}-\epsilon\right)(1+O(1)) \log M\left(\frac{1}{8} M\left(\frac{r}{2^{2}}, f^{[n-2 ; g]}\right)\right.$

$\left.-\left|f^{[n-2 ; g]}(0)\right|, g\right)+O(1)$

$\geq\left(\lambda_{f}-\epsilon\right)(1+O(1))\left[\frac{1}{16} M\left(\frac{r}{2^{2}}, f^{[n-2 ; g]}\right)\right]^{\lambda_{g}-\epsilon}$

$+O(1)$

$$
\begin{aligned}
& \left.\log ^{[3]} M\left(r, f^{[n ; g]}\right)\right) \\
& \geq \log \left(\lambda_{f}-\epsilon\right)+\left(\lambda_{g}-\epsilon\right) \log \left[\frac{1}{16} M\left(\frac{r}{2^{2}}, f^{[n-2 ; g]}\right)\right] \\
& +O(1) \\
& \geq\left(\lambda_{g}-\epsilon\right) \log \left[M\left(\frac{r}{2^{2}}, f^{[n-2 ; g]}\right)\right] \\
& +O(1),
\end{aligned}
$$

Proceeding as above we get

$$
\begin{aligned}
& \log ^{[n]} M\left(r, f^{[n ; g]}\right) \\
& \geq\left(\lambda_{f}-\epsilon\right)(1+O(1)) \log M\left(\frac{r}{2^{n-1}}, g\right) \\
& +O(1), \text { when } n \text { is even }
\end{aligned}
$$

Similarly for odd $n$, we get

$$
\begin{aligned}
& \log ^{[n]} M\left(r, f^{[n ; g]}\right) \\
& \geq\left(\lambda_{g}-\epsilon\right)(1+O(1)) \log M\left(\frac{r}{2^{n-1}}, f\right) \\
& +O(1) .
\end{aligned}
$$

This proves the lemma.

Lemma 2.3 Let $f$ and $g$ be two non-constant entire functions such that $0<\lambda_{f} \leq \rho_{f}<\infty$ and $0<\lambda_{g} \leq$ $\rho_{g}<\infty$. Then for any $\varepsilon\left(0<\epsilon<\min \left\{\lambda_{f}, \lambda_{g}\right\}\right)$,

$$
\begin{aligned}
& \log ^{[n]} \mu\left(r, f^{[n ; g]}\right) \\
& \geq\left(\lambda_{f}-\epsilon\right)(1+O(1)) \log M\left(\frac{r}{2^{n}}, g\right) \\
& +O(1), \text { when } n \text { is even } \\
& \log ^{[n]} \mu\left(r, f^{[n ; g]}\right) \\
& \geq\left(\lambda_{g}-\epsilon\right)(1+O(1)) \log M\left(\frac{r}{2^{n}}, f\right) \\
& +O(1), \text { when } n \text { is odd, }
\end{aligned}
$$

and

for all large values of $r$.

Proof. From (1) we get,

Hence

$$
\mu(2 r, f) \geq \frac{1}{2} M(r, f) .
$$

$$
\log \mu(r, f) \geq \log M\left(\frac{r}{2}, f\right)+O(1) .
$$

The Lemma 2.3 follows from (2) and Lemma 2.2. This proves the lemma.

\section{THEOREMS}

Theorem 3.1 Let $f$ and $g$ be two non-constant entire functions such that $0<\lambda_{f} \leq \rho_{f}<\infty$ and $0<\lambda_{g} \leq$ $\rho_{g}<\infty$. Then for any positive number $A$ and every real number $\alpha$,

(i)

$$
\lim _{r \rightarrow \infty} \frac{\log { }^{[n]} \mu\left(r, f^{[n ; g]}\right)}{\left\{\log \log \mu\left(r^{A}, f\right)\right\}^{1+\alpha}}=\infty
$$

and

(ii) 


$$
\lim _{r \rightarrow \infty} \frac{\log { }^{[n]} \mu\left(r, f^{[n ; g]}\right)}{\left\{\log \log \mu\left(r^{A}, g\right)\right\}^{1+\alpha}}=\infty .
$$

Proof. If $\alpha \leq-1$ then the theorem is trivial. So we suppose that $\alpha>-1$ and $n$ is even. Then from Lemma 2.3 we get for all sufficiently large values of $r$ and any $\varepsilon>0$

$$
\begin{aligned}
& \log ^{[n]} \mu\left(r ; f^{[n ; g]}\right) \\
& \geq\left(\lambda_{f}-\varepsilon\right)(1+O(1)) \log M\left(\frac{r}{2^{n}}, g\right)+O(1) \\
& \geq\left(\lambda_{f}-\varepsilon\right)(1+O(1))\left(\frac{r}{2^{n}}\right)^{\lambda_{g}-\epsilon} \\
& +O(1)
\end{aligned}
$$

Again from Definition 1.1 it follows that for any $\epsilon(>0)$ and for all large values of $r$,

$$
\begin{aligned}
& \left\{\log \log \mu\left(\mathrm{r}^{A}, f\right)\right\}^{1+\alpha} \\
& <\left(\rho_{f}+\varepsilon\right)^{1+\alpha} A^{1+\alpha}(\log r)^{1+\alpha}
\end{aligned}
$$

From (3) and (4) we have for all large values of $r$ and any $\varepsilon\left(0<\varepsilon<\min \left\{\lambda_{f}, \lambda_{g}\right\}\right.$,

$$
\begin{aligned}
& \frac{\log [n]}{\left[\log \log \mu\left(r^{A}, f\right) f^{[n ; g]}\right)} \\
& \geq(1+O(1)) \frac{\left(\lambda_{f}-\varepsilon\right)\left(\frac{r}{2^{n}}\right)^{\lambda_{g}-\varepsilon}+O(1)}{\left(\rho_{f}+\varepsilon\right)^{1+\alpha} A^{1+\alpha}(\log r)^{1+\alpha}} \\
& \geq(1+O(1)) \frac{\left(\lambda_{f}-\varepsilon\right)\left(\frac{r}{2^{n}}\right)^{\lambda_{g}-\varepsilon}}{\left(\rho_{f}+\varepsilon\right)^{1+\alpha} A^{1+\alpha}(\log r)^{1+\alpha}}+o(1) .
\end{aligned}
$$

Since $\varepsilon>0$ is arbitrary, therefore

$$
\lim _{r \rightarrow \infty} \frac{\log { }^{[n]} \mu\left(r, f^{[n ; g]}\right)}{\left\{\log \log \mu\left(r^{A}, f\right)\right\}^{1+\alpha}}=\infty
$$

Similarly for odd $n$ we get

$$
\begin{aligned}
& \log ^{[n]} \mu\left(r ; f^{[n ; g]}\right) \\
& \geq\left(\lambda_{g}-\varepsilon\right)(1+O(1))\left(\frac{r}{2^{n}}\right)^{\lambda_{f}-\epsilon} \\
& +O(1)
\end{aligned}
$$

Hence from (4) and (6) we have the equation (5) for odd $n$.

This proves (i).

The second part of the theorem follows similarly by using the following inequality instead of (4)

$\left\{\log \log \mu\left(\mathrm{r}^{A}, g\right)\right\}^{1+\alpha}<\left(\rho_{g}+\varepsilon\right)^{1+\alpha} A^{1+\alpha}(\log r)^{1+\alpha}$, for all large values of $r$ and arbitrary $\varepsilon>0$. This proves the theorem.

\section{REFERENCES}

[1] D. Banerjee and N. Mondal, Maximum modulus and maximum term of generalized iterated entire functions, Bulletin of the Allahabad Mathematical Society, 27(1) (2012), 117-131.

[2] D. Banerjee and R. K. Dutta, On maximum term of iterated entire functions, The Journal of Indian Academy of Mathematics, 33(1), 2011, 297-308.

[3] J. Clunie, The composition of entire and meromorphic functions, Mathematical essays dedicated to A. J. Macintyre, Ohio Univ. Press, (1970), 75-92.

[4] R. K. Dutta, Further growth of iterated entire functions in terms of its maximum term, International journal of Nonlinear Analysis and Applications, 2(2), 2011, 86-91.

[5] R. K. Dutta, Growth of iterated entire functions in terms of its maximum term, Acta Universitatis Apulensis, 30, 2012, 209-219.

[6] W. K. Hayman, Meromorphic Functions, The Clarendon Press, Oxford, 1964.

[7] D. Sato, On the rate of growth of entire functions of fast growth, Bull. Amer. Math. Soc., 69 (1963), 411-414.

[8] A. P. Singh, On maximum term of composition of entire functions, Proc. Nat. Acad. Sci. India, 59(A), (1989), 103-115.

[9] G. Valiron, Lectures on the general theory of integral functions, Chelsea Publishing Company (1949)

[10] C. C. Yang and H. X. Yi, Uniqueness theory of meromorphic functions, Kluwer Academic Publishers and Science Press, Beijing, 2003. 